\title{
Image De-noising Based on Mixed-domain Algorithm
}

\author{
Ting Yan, Liejun Wang ${ }^{*}$, Jiaxing Wang \\ College of Information Science and Engineering, Xinjiang University, Urumuqi 830046, China \\ yant210@sina.cn, *wljxju@xju.edu.cn
}

\begin{abstract}
Aiming at images contain a large number of gaussian noise, edge blur etc, the paper proposes an improved fuzzy-shrink denoising algorithm, which is in a mixed-domain. The results show that the algorithm not only have optimal de-noising effect, and also the processed image is closer to the original image on the visual effects.

Index Terms - image de-noising, improved fuzzy-shrink algorithm, mixed-domain, visual effects
\end{abstract}

\section{Introduction}

For image imaging and transmission process susceptible to noise, which lead to the image quality degradation and poor visual effects, researchers have proposed many image denoising methods.Reference[1]proposed a hybrid filter denoising method, but in severe noise pollution, it is not obvious on de-noising and protection of image edge details;Reference[2] proposed hidden Markov tree model denoising algorithm. However, the time complexity of the algorithm is too high, and can not effectively remove noise; Reference[3]Image de-noising based on classification standard in non-subsampled Contour-let transform domain, although it has higher peak signal to noise ratio(PSNR), and good visual effect, it requires a high time complexity.Reference[4] used the dual-tree complex wavelet transform de-noising algorithm, the transformation has a good direction and translation invariance. With the extensive application of wavelet transform, its limitations that lack of multidirectional selection and non-sparse are also increasingly apparent. In 2002,Do and Martin Vetterli have proposed an image representation method-Contour-let transform, which has these characteristics of multi-resolution, localization, multi-direction[5]. It is a twodimensional transform, the final result use the base structure that similar to contour (contour segment) to approximate the original image, and the length ratio of its base function support interval is changed with the scale changes, and in a way which can be close to the optimal approach to redundantly describe the image details such as edges and textures, but Contour-let itself is lack of translation invariance, therefore on this basis, Cunha and others MNDO proposed its down sampling form, that is the non-sampled Contour-let transform (NSCT) [6].

In this paper, for correlation coefficients of noise is weak or irrelevant in NSCT domain [7], but it has a strong correlation between the scale and scale. Fuzzy feature is proposed to simulate scales relevance of NSCT coefficient in order to distinguish image detail and noise in each sub-band of NSCT domain, then use the fuzzy membership degree rule to filter noise. The method use images of different Gaussian noise variance to conduct simulation experiment, qualitatively and quantitatively analysize and compare the proposed method fuzzy-shrink (fuzzy-shrink using NSCT) with fuzzy-shrink (fuzzy-shrink using DWT) de-noising method, ant-shrink (antshrink using DWT) de-noising and sure-let (sure-let using DWT) de-noising methods. The Simulation results show that the proposed method are significantly better than the above three methods in PSNR, mean square error (MSE), and get a better visual effect.

\section{Based on Improved Fuzzy-Shrink Image De-Noising}

\subsection{Fuzzy characteristic structure based on the neighborhood information of NSCT domain}

Image linear transformation redundancy expression and translation invariance are two important factors in the image transform domain.First of all, two-dimensional discrete wavelet transform (Discrete Wavelet Transform, DWT) can not achieve optimal approximation when represents the opposite sex edges and texture,secondly,DWT transform will produce pseudo-Gibbs phenomena in processing singularity with threshold,this is because of the translation invariance of wavelet transform[8]. Aiming at the problem mentioned above, the paper improved fuzzy-shrink(DWT) algorithm[9], and apply fuzzy-shrink algorithm to NSCT domain,the multi-scale and multi-direction of NSCT transform can flexibly capture details of the images, and has rich image details and edge information in the image redundancy expression, which is beneficial to construct fuzzy feature; and NSCT transform has translational invariance, which will not arouse image distortion that produced by pseudo-Gibbs phenomenon in using threshold to process image singularity.

The NSCT coefficients of image are not independent, but have a certain correlation[7].After NSCT transform, the energy of image edge coefficients is concentrated and amplitude is large,then will get a large areas of similarity, while the noise energy dispersion and the amplitude is small,then the similarity of region coefficient is small.Taking into account this dependence of NSCT coefficients, using larger similarity coefficient neighborhood to give greater weights, for the smaller one give smaller weights. Therefore, fuzzy neighborhood similarity function $\mathrm{m}(1, \mathrm{k})[9]$ and the similarity ambiguity functions $(1, \mathrm{k})[9]$ of spatial position are defined as follows:

$$
\begin{gathered}
m(l, k)=\exp \left(-\left(\frac{y_{s, d}(i, j)-y_{s, d}(i+l, j+k)}{T h r}\right)^{2}\right) \\
s(l, k)=\exp \left(-\left(\frac{l^{2}+k^{2}}{N}\right)\right)
\end{gathered}
$$

Where, $Y_{s, d}(i, j)$ is coefficient of position $(i, j)$ of scale $s$ and direction sub-band $d$ in the NSCT transform domain, $y_{s, d}(i+1$, $\mathrm{j}+\mathrm{k})$ is coefficient of position $(\mathrm{i}+1, \mathrm{j}+1)$ of scale $\mathrm{s}$ and 
direction sub-band $d$ in the NSCT transform domain, which is represented each coefficient with $\mathrm{y}_{\mathrm{s}, \mathrm{d}}(\mathrm{i}, \mathrm{j})$ as center within local neighborhood window. $T h r$ is the threshold that have relation with noise variance estimation $\sigma_{\mathrm{n}}$, Thr value can be obtained from the following formula(3), $\sigma_{n}$ using robust median estimation[10], $\mathbf{y}_{\mathbf{s}, \mathbf{d}}$ is the NSCT coefficient matrix in scale $\mathrm{s}$ and direction $\mathrm{d}, \mathrm{c}$ ranges from 2 to 3.In the experiments, when the value of $\mathrm{c}$ is 2 , the effect will be the best .1 and $\mathrm{k}$ can determine the size of neighborhood window, 1 belongs to interval $[-\mathrm{L} \ldots \mathrm{L}]$, $\mathrm{k}$ belongs to $[-\mathrm{K} \ldots \mathrm{K}]$, for example: when 1 is $2, \mathrm{k}$ is 2 , the size of neighborhood window is $5 \times 5$, then the adaptive weights $\mathrm{w}(1, \mathrm{k})$ [9] which is adapt to every NSCT coefficient can be constructed by these two similarity ambiguity function, defined as follow formula (5):

$$
\begin{aligned}
& \text { Thr }=c \sigma_{n} \\
& \sigma_{n}=\frac{\operatorname{Median}\left(\left|y_{s, d}\right|\right)}{0.6745} \\
& w(l, k)=m(l, k) \times s(l, k)
\end{aligned}
$$

Finally, according to the weights for each sub-band NSCT construct the coefficients within the fuzzy eigenvalues $f(i, j){ }^{[9]}$, is defined as follows:

$$
f(i, j)=\frac{\sum_{l=-L}^{L} \sum_{k=-K}^{K} w(l, k) \times\left|y_{s, d}(i+l, j+k)\right|}{\sum_{l=-L}^{L} \sum_{k=-K}^{K} w(l, k)}
$$

According to literatures that local means can be used to threshold shrinkage function [9], and the main differences of fuzzy characteristics and local means is that the fuzzy feature can effectively distinguish edge structure coefficients and noise coefficients[9], so this feature is used to construct fuzzy threshold shrinkage function in the paper.

\subsection{NSCT domain coefficients contraction rules based on fuzzy feature}

Shrink the image coefficients of different directional subband which is decomposed by NSCT, typically follow the basic principle: reducing the coefficients of the area which contains noise to zero, and retaining coefficients of the area which contains the edge and details as much as possible[9], this paper uses a fuzzy rule-based fuzzy feature to shrink NSCT coefficients.

Fuzzy logic idea is put forward by Zadeh and it is widely used in various fields[11]. Fuzzy sets and fuzzy membership function can be understood as fuzzy rules based on causal system[12], when the fuzzy feature is known, IF-THEN structure need to be constructed only to shrink NSCT coefficient, that is: if the fuzzy feature $f(i, j)$ values is large, then each sub-band coefficient $y_{s, d}(i, j)$ of NSCT can be shrunk in a small range to retain more details and edge information of the image, and that fuzzy feature indicates the contractive law of sub-band NSCT coefficients of noise. and fuzzy membership function can be seen as a curve, this curve defines how the point value of the input space mapped to the value of membership in the range zero to one, the membership function is usually shown with $u$, input space is discussion domain, the function itself can be arbitrary, but it must meet the function value in the range zero to one. Therefore, the membership function can be a piecewise linear function, gaussian distribution function, sigmoid function, quadratic or cubic polynomial functions etc. This paper refers to the spline curve[9], its shape is s-shaped, function expression is as follows:

$$
u(x)=\left\{\begin{array}{l}
0, x \leq T_{1} \\
2 \times\left(\frac{x-T_{1}}{T_{2}-T_{1}}\right)^{2}, T_{1} \leq x \leq \frac{T_{1}+T_{2}}{2} \\
1-2 \times\left(T_{2}-\frac{x-T_{1}}{T_{2}-T_{1}}\right)^{2}, \frac{T_{1}+T_{2}}{2} \leq x \leq T_{2} \\
1, x \geq T_{2}
\end{array}\right.
$$

$\mathrm{T} 1$ and $\mathrm{T} 2$ in which the parameters for the slope of the curve portion of the extreme point value, $\mathrm{x}$ is the independent variable curve, in this article is the fuzzy eigenvalues $x \mathrm{f}(\mathrm{i}, \mathrm{j})$, $\mathrm{T} 1$ and $\mathrm{T} 2$ and the estimated noise variance is on-related parameters, in order to verify that $\mathrm{T} 1$ and $\mathrm{T} 2$ and $\sigma_{\mathrm{n}}$ relationship, the paper take a lot of pictures of different noise variance test verifies available $\mathrm{T} 1, \mathrm{~T} 2, \sigma_{\mathrm{n}}$ satisfy the following equation [9]:

$$
\begin{aligned}
& T_{1}=K_{1} \times \sigma_{n} \\
& T_{2}=K_{2} \times \sigma_{n}
\end{aligned}
$$

Where $\mathrm{K} 1$ and $\mathrm{K} 2$ are constants variable, the present results show that, When $\mathrm{K} 1=3 \mathrm{~K} 2=2$, its de-noising effect is the best.

\section{Description of Algorithm Process}

In summary, the proposed image de-noising method of the combination of NSCT and fuzzy theory steps are as follows:

(1)Non-subsampled Contour-let transform(NSCT) for the noisy image .

(2)Estimate noise variance $\sigma_{\mathrm{n}}$ of the NSCT coefficients for each sub-band according to the method of section 2.2 (4) .

(3)Take the window size of $5 \times 5$, neighborhood similarity function and fuzzy spatial similarity ambiguity function of each direction and each scale coefficients of NSCT domain can be obtained according to formula 2.2 (1),(2),(3), then each adaptive weights of NSCT coefficient can be got from formula (5), finally by the formula(6) fuzzy feature value of each coefficient of each sub-band can be obtained.

(4)According to 2.3(7), (8), (9) obtain each fuzzy membership function value $\mathrm{u}(\mathrm{f}(\mathrm{i}, \mathrm{j}))$ of fuzzy characteristic values of each sub-band coefficient, finally, process each NSCT coefficients as following formula:

$$
x(\hat{i, j})=u(f(i, j)) \times y_{s, d}(i, j)
$$

(5) Conduct NSCT inverse transform using the coefficients $x(\hat{i}, j)$ which is processed, and reconstruct image,thus de-noised images can be obtained. 


\section{Experimental Results and Analysis.}

In this paper, with 2 GB memory of Intel processor, images which has a size of $512 \times 512$ and 256 gray scale served as a test sample to verify the correctness and effectiveness of the proposed algorithm. Added gaussian white noise of zero mean and different variance to the original image, and compare fuzzy-shrink (DWT) de-noising method [9], antshrink (DWT) de-noising method [13], sure-let (DWT) denoising method [14] with the proposed method, the results of the four methods can be measured by objective evaluation using peak signal to noise ratio (PSNR) and mean square error(MSE).

$$
P S N R=10 \log \left(255^{2} / M S E\right)
$$

Where

$$
M S E=(M \times N)^{-1} \sum_{i=1}^{M} \sum_{j=1}^{N}(g(i, j)-h(i, j))^{2}
$$

Where $g(i, j)$ denotes the noise free image and $h(i, j)$ denotes the de-noised image. $\mathrm{M}$ and $\mathrm{N}$ are the length and width of the image.

NSCT decomposition stages is three in this experiment, the first layer is divided into 16 directions, the second layer is divided into eight directions, and the third layer is divided into four directions. The DWT wavelet basis is "sym8", decomposition scale are four scales. Table 1 is the results of peak signal to noise ratio (PSNR) and mean square error(MSE) of the image of adding different noise variance Gaussian white noise. Figure 2 is the images of using different ways to filter noise, which is add gaussian white noise of a standard deviation of 30 .

To verify the validity of the proposed algorithm, a large number of images are chosen as samples. There have two typical images and its data shown below.

As can be seen from Table 1, with the noise variance ranges from 0.01 to 0.1 , the PSNR values are decreasing, while the MSE values are increasing. When the noise variance is 0.01 , the PSNR values respectively rise 4.37 percent in antshrink (DWT) algorithm, 3.66 percent in sure-let(DWT) algorithm,3.98 percent in fuzzy-shrink(DWT) algorithm and 8.99 percent in the proposed algorithm. The same way to the MSE values of the remote sensing images, when the noise variance is 0.1 , the MSE values respectively decrease 20.06 percent in ant-shrink(DWT) algorithm,33.98 percent in surelet(DWT) algorithm,40.15 percent in fuzzy-shrink(DWT) algorithm, and 56.68 percent in the proposed algorithm. In all simulations, as opposed to the other three methods, the proposed method is of the highest PSNR, and MSE is minimal.

As can be seen from Figure 1, the de-noising method used discrete wavelet transform (DWT) is slightly less in decreasing image noises than the results of de-noising algorithm of this paper. To get an obvious results on preserving image edges and details, remote sensing images are chosen to test. As can be seen from Figure 2, the results of denoising algorithm of this paper in preserving image edges and details is better than the de-noising method used discrete wavelet transform (DWT), and have a relatively good visual effect, not only smooth the noise and also maintain a clearer image texture.

Tab.1 The resulting PSNR and MSE values of two images (Barbara and Remote sensing image) after adding different white noises with different

\begin{tabular}{|c|c|c|c|c|c|c|c|}
\hline Images & $\begin{array}{c}\text { noise } \\
\text { variance }\end{array}$ & index & $\begin{array}{l}\text { noisy } \\
\text { image }\end{array}$ & $\begin{array}{c}\text { Ant- } \\
\text { shrink } \\
(\mathrm{DWT}) \\
\end{array}$ & $\begin{array}{l}\text { Sure-let } \\
\text { (DWT) }\end{array}$ & $\begin{array}{c}\text { Fuzzy- } \\
\text { shrink } \\
\text { (DWT) } \\
\end{array}$ & proposed \\
\hline \multirow{6}{*}{ Barbara } & 0.01 & PSNR & 28.14 & 29.37 & 29.17 & 29.26 & 30.67 \\
\hline & & MSE & 646.80 & 410.36 & $258 . .73$ & 192.02 & 85.06 \\
\hline & 0.05 & PSNR & 18.57 & 24.12 & 23.15 & 23.08 & 24.73 \\
\hline & & MSE & 2796 & 1969.4 & 1265.5 & 669.7 & 491.8 \\
\hline & 0.1 & PSNR & 14.16 & 19.97 & 18.57 & 19.26 & 21.55 \\
\hline & & MSE & 4624.6 & 3869.1 & 3089.5 & 2356.4 & 1447.1 \\
\hline \multirow{6}{*}{$\begin{array}{c}\text { Remote } \\
\text { sensing } \\
\text { image }\end{array}$} & 0.01 & PSNR & 22.10 & 24.66 & 25.07 & 24.58 & 26.47 \\
\hline & & MSE & 1035.9 & 852.64 & 569.7 & 307.54 & 261.85 \\
\hline & 0.05 & PSNR & 16.07 & 21.34 & 20.17 & 20.32 & 22.77 \\
\hline & & MSE & 3582.1 & 2138 & 1959.6 & 1164.05 & 875.3 \\
\hline & 0.1 & PSNR & 11.98 & 15.36 & 15.09 & 16.73 & 19.85 \\
\hline & & MSE & 4584.52 & 3664.9 & 3026 & 2743.57 & 1983.29 \\
\hline
\end{tabular}
standard deviations and de-noising by different algorithms

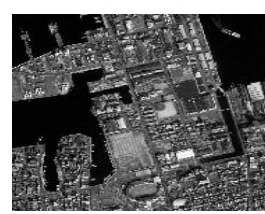

(a)Original Image

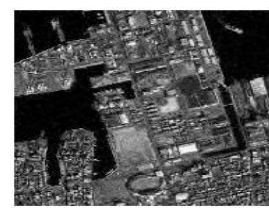

(d) Ant-shrink(DWT)

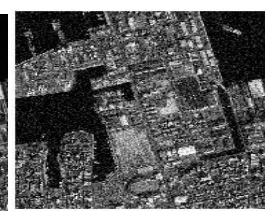

(b)Noisy Image

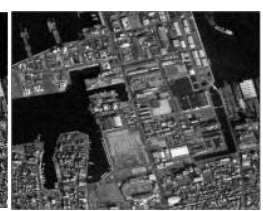

(e)Fuzzy-Shrink(DWT)

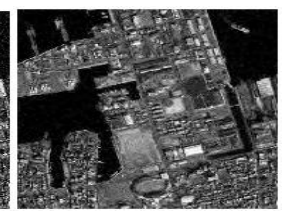

(c)Sure-let (DWT)

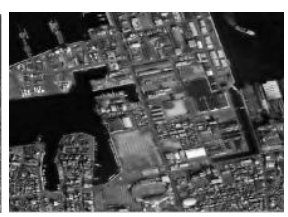

(f) Proposed
Fig.1 Using four kinds of de-noising method for noisy image Barbara

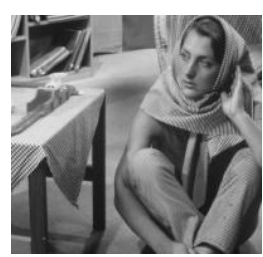

(a)Original Image

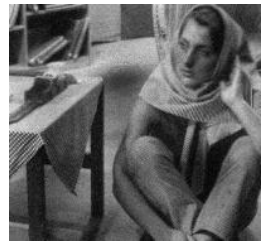

(d) Ant-Shrink(DWT)

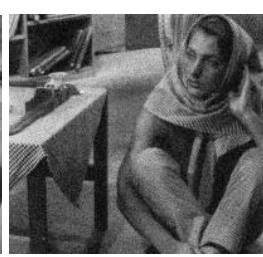

(b)Noisy Image

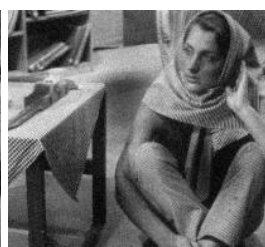

(e)Fuzzy-Shrink(DWT)

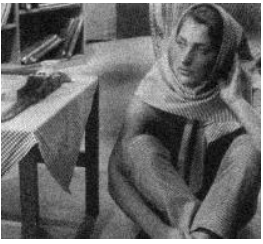

(c)Sure-let (DWT)

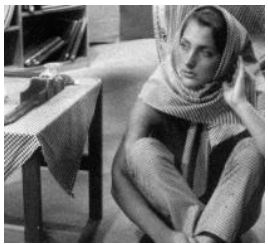

(f) Proposed
Fig.2 Using four kinds of de-noising method for noisy remote sensing image 
In summary, the proposed algorithm has certain advantages in de-noising image compared to other de-noising algorithm and has shown a better performance. The reasons are as follows:

(1) Use non-subsampled Contour-let Transform(NSCT). Compared to DWT, at first ,the multi- scale and multidirection of NSCT can flexibly capture detail information of images, and have rich image details and edge information in the expression of the image redundancy, which is beneficial to construct fuzzy features, thus making this method get a better result than fuzzy-shrink (fuzzy-shrink using DWT)de-noising method[9], ant-shrink (ant-shrink using DWT)de-noising method[13] and sure-let(sure-let using DWT) ${ }^{[14]}$ de-noising method. Secondly, NSCT has translational invariance, which will not occur pseudo-Gibbs phenomenon when using threshold to process image singularity, this is better than the de-noising results of the two-dimensional discrete wavelet transform, and effectively maintain the direction and detail information of the original image. It is because of the multiscale, multi-directional, good spatial, frequency domain location, and the ability to effectively capture the image geometry information of NSCT transform is superior to wavelet transform, so NSCT is chosen in this paper.

(2) Use fuzzy theory. Based on fuzzy modeling of the coefficients correlation in scales of NSCT domain, and use neighborhood similarity function and spatial ambiguity function of each scale and each direction coefficient to construct adaptive fuzzy similarity weights, thus getting fuzzy feature values of each coefficient. Finally use the fuzzy contraction rules of fuzzy membership function to shrink each fuzzy eigenvalues. Select fuzzy eigenvalues for shrink is because fuzzy feature can effectively distinguish the part of edge structure coefficients and coefficients belonging to the noise, for maintaining image detail is superior than ant-shrink (DWT)de-noising method[13], sure-let (DWT) algorithm[14].

(3) Use the adaptive weights.In the process of fuzzyShrinking, the structure of neighborhood window is used for constructing fuzzy feature, it combines neighborhood information of NSCT coefficient, and considers the spatial location of coefficients, then constructs adaptive weight structure, and further improves the PSNR of the de-noising image, while other three kinds of algorithm does not have selfadaptivity.

Although higher peak signal to noise ratio (PSNR), lower mean square error (MSE) and good visual effects can be got with the proposed algorithm, its time complexity is a little high.

\section{Conclusions}

This paper proposes an improved fuzzy-shrink de-noising algorithm, which combined the fuzzy domain and transform domain, apply the fuzzy-shrink algorithm to NSCT domain, use fuzzy theory to conduct correlation model of the scale coefficient of non-subsampled Contour-let transform(NSCT), and in the basis of neighborhood information and spatial location information of the NSCT coefficients to construct neighborhood similarity ambiguity function and spatial similarity ambiguity function, then get adaptive weights and use it to construct the fuzzy feature values, at last regard it as vectors of fuzzy shrink function to conduct de-noise. Simulation results show that the proposed algorithm can get higher peak signal to noise ratio (PSNR) and lower mean square error (MSE) in de-noising image and have a better visual effect. Although it has advantages in de-noising and preserving image edges and details, its time complexity is high, thus can bring difficulty to its application. The next step will be in the direction of improve the time complexity meanwhile maintaining the original advantage on the basis of the paper.

\section{Acknowledgment}

The research work was supported by National Natural Science Foundation of China under Grant No. 61261036.The authors are grateful for the helpful suggestions and comments provided by the anonymous reviewers of this work.

\section{References}

[1] Xiang Liling, "Method of Video Image De-Noising Based on Mixed Filter" Journal of Jilin University(Information Science Edition), vol.31, no.3,pp.2826-2837,2013

[2] Crouse M S, Nowak R D, Baraniuk R G, "Wavelet-based statistical signal processing using hidden Markov models" IEEE Transaction on Signal Processing,vol.46,no.4,pp. 886-902,1998

[3] Sha Hao, "Image de-noising based on classification standard in nonsubsampled Contour-let transform domain" Journal of Hefei University of technology,vol.36,no.7,pp. 7040-7049,2013

[4] Kings-bury N G, "The dual-tree complex wavelet transform:a new technique for shift invariance and directional filters" IEEE Digital Signal Processing Workshop,DSP98, Bryce Canyon,vol.86,1998

[5] Mich N.Do, M N,VETTERLI M, "The Contour-let Transform: An Efficient Directional Multi-resolution Image Representation"IEEE Transactions on Image Processing,vol.14,no.12,pp.2091- 2106,2005

[6] Cunha A L da,Zhou J P, and Do M N. "The Non-subsampled Contour-let transform :Theory,Design and Application" IEEE Transactions on Image Processing.vol.15,no.10,pp.3089-3101,2006

[7] Yan Sheng, Liu Minggang, Yuan Jianping, Hou Chaohuan, "Novel ultrasound image de-noising method based on NSCT transformation"Beijing:Chinese Journal of Science Instrument,vol.33,no.5,pp.1005-1012,2012

[8] Yu Du-fa, Shao Jian hua, "Research on Image Denoising Based on Wavelet Adaptive Threshold" Computer technology and development. vol.23,no.8, pp.251-253,2013

[9] Jamal Saeedi, Mohammad Hassan Moradi “'A New Wavelet-based Fuzzy Single and Multi-channel Image De-noising"Image and Vision Computing, vol.28,pp.1611-1623,2010

[10]J.Jennifer Ranjani,S.J.Thiruvengadam,"Generalized SAR Despeckling Based on DTCWT Exploiting Interscale and Intrascale Dependences" IEEE Geoscience And Remote Sensing Letters,vol.8,no.3,pp.552556,2011

[11]S.M. Saad.,"Application of fuzzy logic and genetic algorithm in biometric text-independent writer Identification,"IET Information Security.vol.5, no.1,pp.1-9,2011

[12]Qiang Zhenping, Di Guangzhi, Chen Xu, "Land use classification of remote sensing images fuzzy rules based on fuzzy subsets" Remote Sensing Technology and Application,vol.28,no.4,pp.633-639 2013

[13]Jing Tian,Weiyu Yu,Lihong Ma."Ant-shrink:Ant colony optimization for image shrinkage,'Pattern Recognition Letters.vol.31,pp.1751-1758,2010

[14]F. Luisier, T. Blu, "SURE-LET multichannel image de-noising: inter-scale orthonormal wavelet Thresholding," IEEE Transaction.Image Process. vol.17, no.4,pp.482-492,2008 